\title{
A User Mobility-Pattern-Based Location Strategy for Next Generation Wireless Multimedia Networks
}

\author{
Wenchao Ma \\ Department of Electrical and \\ Computer Engineering \\ University of Florida \\ Email: mawench@ufl.edu
}

\author{
Yuguang Fang \\ Department of Electrical and \\ Computer Engineering \\ University of Florida \\ Email: fang@ece.ufl.edu
}

\begin{abstract}
For a wireless network to effectively deliver services to the mobile users, it must have an efficient way to keep track of them. For the next generation wireless multimedia networks which can provide wide bandwidth services, the radio resource becomes more competitive. Many strategies have been proposed to reduce the spectrum consumption of the users location update and paging messages, such as the PBS scheme. In this paper, we study the PBS scheme performance under various conditions by simulations. We also propose a new scheme - MPBS which includes user time information in the profile. The user location is computed by the system based on not only the distribution probability but also the system current time. The simulation results suggest that the MPBS scheme can generate far less signaling traffic and paging delay than the PBS scheme does.
\end{abstract}

\section{INTRODUCTION}

The characteristic of the third generation wireless communication systems is that both voice and data service are provided. The mobility management strategy for $3 \mathrm{G}$ systems is trying to meet the data service requirements and making users belonging to different $2 \mathrm{G}$ networks can roam to each other ( [1], [2]). In order to track the users, the system service areas are partitioned into cells, and the cells are usually aggregated into groups (LAs) geographically. To deliver services to a user, all the cells in the LA covering that user will be paged to establish the radio link connection. For next generation systems, the cells are partitioned into routing areas (RAs). An RA is typically a subset of an LA. The small size of cell group can facilitate the system to provide users data service with required quality. However the location update messages sent by users increase with the reducing registration area size. Since the radio bandwidth is considered the scarcest resource, a lot of research has been carried out to minimize the impact of the user location management traffic on systems ( [3], [4]). In future generation systems, the services should be user-oriented, namely, the networks can provide different kinds of services for different users. In order to provide user-oriented service, the system needs to store the user profile which records the necessary information. Based on this consideration, a profilebased location strategy (PBS) was proposed in [5] and [6]. In the PBS scheme, the system records each user's most likely itinerary. In this paper, we study the relationship between the PBS scheme performance and the user call-to-mobility ratio
(CMR) by simulations. In [7], we proposed a new mobility management strategy - user mobility-pattern-based location scheme (MPBS). In the MPBS scheme, the time a user enters a location area and the residence time in that location area are also recorded in the user's profile. The location area number needed to be paged when a call arrives is decided by the current system time and the user states. In this paper, we further study the MPBS scheme performance by simulations, The results show that the MPBS can achieve much better performance than PBS strategy under various conditions.

The paper is organized as follows. In section II, we introduce the PBS and MPBS schemes in detail. We analyze the scheme performance costs in section III. Section IV gives the simulation results. In section V, we have the conclusions.

\section{PBS SCHEME AND MPBS SCHEME}

The PBS scheme is proposed in [5] and [6], which can effectively reduce the user update cost. But this scheme reduces the update cost at the expense of increasing paging cost. The MPBS scheme is proposed in [7] and proved by simulations that it can achieve better performance under wider conditions.

\section{A. Profile-based location scheme (PBS)}

In PBS scheme, the system maintains the records of each user's most likely itinerary. The probability distribution of a user's location is known already. It can be provided by the mobile terminal or estimated by the system using the user's past calling history.

In the scheme, if $A_{i}$ is one of the location areas in the record list, the user's most likely itinerary can be defined as $\left\{A_{i}\right\}_{i=1}^{k}$, where $k$ is the element number in the set. The probability of a user being in a location area $A_{i}$ is $\alpha_{i}$. The system maintains a list of $\left(A_{i}, \alpha_{i}\right)$ pairs for some time interval $T$. The probability of a user being within $\left\{A_{i}\right\}_{i=1}^{k}$ is given as $\kappa=\sum_{i=1}^{k} \alpha_{i}$. If $\kappa<1$, there is always a probability that the user can not be found in the list. If the user follows his/her daily itinerary strictly, namely, the user keeps roaming in $\left\{A_{i}\right\}_{i=1}^{k}$, no registration is needed. When the user is out of the list, the mobile terminal is required to manually register to the system. So the mobile terminal must keep a copy of the list. If a call arrives for a user, the location areas that the user 


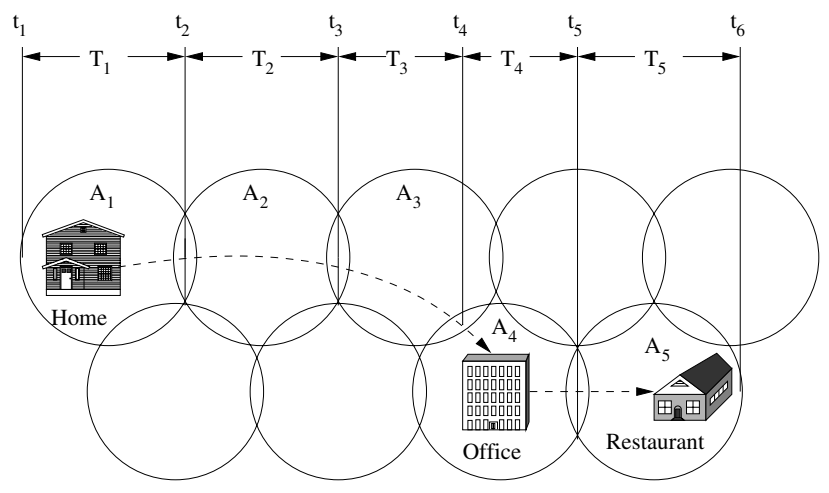

Fig. 1. Mobility pattern based scheme procedures

could be in are paged in the descending order of $\alpha_{i}$ until the user is found. The PBS scheme can reduce the location update cost effectively at the expense of increasing the total paging cost or paging delay. In [6], the total paging cost was derived, given three known probability distributions, by the expected location area numbers needed to be paged before the user can be found. The details about the PBS scheme can be found in [6]. In fact, it is intuitive that the total cost of PBS scheme has tight relationship with the user's CMR. In this paper, we study the CMR effects on PBS scheme under various conditions.

\section{B. Mobility-pattern-based scheme (MPBS)}

In order to improve the system performance, we proposed MPBS scheme in [7]. The MPBS strategy can reduce the user update cost and try to limit the total paging cost at the same time. In this scheme, only two more elements, the time a user enters $A_{i}\left(t_{i}\right)$ and the residence time in $A_{i}\left(T_{i}\right)$, are added in the user profile. The HLR will keep a list of 4tuple $\left(A_{i}, t_{i}, T_{i}, \alpha_{i}\right)$ for each user. Fig.1 shows an example of the MPBS scheme. If a user lives in location area $A_{1}$ and works in his office building which is located in $A_{4}$. Before the user reaches his office, he will pass through $A_{2}$ and $A_{3}$; The user also likes to have his lunch break in a restaurant which is in $A_{5}$. If the user follows the itinerary everyday and the information is stored in his profile. The network can locate the user based on the profile and current system time. We assume the cardinality is $k$. In the list, the tuples are not ordered according to $\alpha_{i}$ but to $t_{i}$. We define a user's daily routine to be the user mobility pattern (UMP) for that user. In reality, a mobile user could deviate from his/her daily route for some traffic or other reasons so that the actual route may be different from the UMP. So, we define the user's actual path information as the User Actual Path (UAP). The mobile user's state can be determined by comparing the similarity between UMP and UAP. If the user follows the profile strictly, we say the user follows the time-sequence pattern; if the user just follows the location areas order but not the time information in the profile, we define the user follows the sequence pattern. The exact definitions about the two patterns can be found in [7]. In MPBS scheme, a user can be in one of the four states at a time:
State 1: if a user follows the time-sequence pattern, we define the user in state 1.

State 2: if a user follows the sequence pattern, we define the user in state 2 .

State 3: if a user enters $A$ and does not follow any of the above two patterns but $A \in\left\{A_{i}\right\}$, we define the user in state 3.

State 4: if $A \notin\left\{A_{i}\right\}$, we define the user in state 4 .

When a call arrives for a user, the system can locate the user by different paging strategies for different states. There are many mechanisms to decide the user's states. In [7], we employed edited distance to determine the user states by defining three operations: inserting, deleting and changing. Every operation is assigned a weight. The detail procedures can be found in [7]. In MPBS scheme, when the user is in state 1,2 or 3 , we say the user is in-the-pattern, otherwise the user is out-of-pattern. we define $\varphi$ the user out-of-pattern probability and $\varphi=1-\sum_{i=1}^{k} \alpha_{i}$. The user is in state 4 when the mobile terminal is out-of-pattern. A user in the MPBS scheme needs to register with the system when he/she switches from the in-the-pattern to out-of-pattern or vice versa. When the user is out-of-pattern, a registration message is sent to the system to update his/her location every time the user enters a new location area, just as the IS-41 or GSM MAP scheme. When the user is in-the-pattern, the user may need to send state update message to the system. The state update message is different from the registration message, it informs the HLR the state change. No registration or state message needs to be sent if the user keeps the state unchanged. When a call arrives, the HLR can get the user's state information and adopt different paging strategies based on it. If the user is in state 1 , the system can know which location area the user is in according to the current system time and page it. There is the probability that the user just moves out of the being paged area and enters the next one. If the user does not change state, only the next location area needs to be paged too. If the user is in state 2 , the system can know the location areas the user is not in and only needs to page the subsequential areas in the descending order of $\alpha_{i}$. If the user is in state 3 , the user will be paged according to $\alpha_{i}$, just like the PBS scheme. If the user is out-of-pattern, namely in state 4, the system knows the user's exact location and pages the respective location area.

As we can see from the three schemes, If we treat the state update message and the registration message same, the IS-41 or GSM MAP schemes will generate the most update messages and the PBS scheme will generate the least ones. The MPBS is in the middle of them. Although the MPBS scheme may generate more update messages than the PBS scheme does, it will reduce the total paging cost dramatically and achieve total cost saving greatly. We can see that in the section III.

\section{COST EVALUATION}

In this section, we define $C_{u}$ the cost for location registration and $C_{p}$ the cost for paging one location area. In our analysis, we do not consider the call delivery processing cost because it is same for all the three schemes. 
For IS-41/GSM MAP scheme, the total cost between two consecutive calls can be given as below ( [7])

$$
C_{G S M}=\frac{C_{u}}{\rho}+C_{p},
$$

where $\rho$ is the CMR. In the following analysis, we assume there are $k$ location areas in the user's profile. For the PBS scheme, we define $E(k)$ the average location area number that has to be paged before the user is found. So it is straightforward that the total cost for PBS scheme is

$$
\begin{aligned}
C_{P B S} & =\omega_{1} \frac{C_{u}}{\rho}+\kappa E(K) C_{p}+(1-\kappa) C_{p} \\
& =\omega_{1} \frac{C_{u}}{\rho}+[\kappa(E(k)-1)+1] C_{p},
\end{aligned}
$$

where $\omega_{1}$ is the probability that the user moves in or out of the profile between two consecutive call arrivals in the PBS scheme. The analysis of the total cost for MPBS is more complicated. If we define $C_{p_{i}}$ the paging cost for a user in state $i$ and $\pi_{i}$ the probability that the user is in state $i$ when a call arrives, respectively; the total cost of MPBS can be expressed:

$$
C_{M P B S}=\omega_{2} \frac{C_{u}}{\rho}+\sum_{i}^{4} \pi_{i} C_{p_{i}},
$$

where $\omega_{2}$ is the probability of the user's movements that need to update to the HLR. It is obvious that $\sum_{i}^{4} \pi_{i}=1$.

We need derive $C_{p_{i}}$ to specify the total cost of MPBS scheme. In the MPBS scheme, when a user is in state 1, if a call arrives, the location area the user is currently in will be paged according to the profile. However, there is the probability that the user just moves into the next location area when the current location area is being paged. In order to make sure the user can be found, the next location area needs to be paged if no response is received in the predefined time in current area. If the user enters location area $A_{i}$ at $t_{\text {actual }}$ and the recorded time is $t_{i}$ in the user profile. We assume that $t_{\text {actual }}-t_{i}$ follows Gaussian distribution with zero mean and variance $\sigma . T_{\text {threshold }}$ is defined to be the time threshold. If $\left|t_{\text {actual }}-t_{i}\right|>T_{\text {threshold }}$, we say the user is not in state 1 anymore. Then the probability that a user just moves into next location area when a page message arrives is $0.5-Q\left(\frac{T_{\text {threshold }}}{\sigma}\right)$, where $Q(\cdot)$ is the $Q$ function. Since the paging expiration time is very short, we ignore the probability that a user crosses more than one location areas when a paging message arrives. Based on the above results, we can obtain

$$
\begin{aligned}
C_{p_{1}} & =C_{p}+\left[0.5-Q\left(\frac{T_{\text {threshold }}}{\sigma}\right)\right] C_{p} \\
& =\left[1.5-Q\left(\frac{T_{\text {threshold }}}{\sigma}\right)\right] C_{p} .
\end{aligned}
$$

If a user is in state 2 when a call arrives, only the following location areas need to be paged because it is known that the user is not in the first $m(m<k)$ location areas. So the distribution probability in the following location areas is conditional. If we assume there are $m$ location areas has been crossed before a call arrives, and let $X_{m}=\alpha_{1}+\alpha_{2}+\cdots+\alpha_{m}$ and $k^{\prime}=k-m$, we can get the conditional probability distribution for the next $k^{\prime}$ location areas as $\alpha_{1}^{\prime}=\frac{\alpha_{m+1}}{1-X_{m}}$, $\alpha_{2}^{\prime}=\frac{\alpha_{m+2}}{1-X_{m}} \cdots, \alpha_{k^{\prime}}^{\prime}=\frac{\alpha_{k}}{1-X_{m}}$ respectively. If the average paged number is $E\left(k^{\prime}\right)$, the $C_{p_{2}}$ can be written as

$$
C_{p_{2}}=E\left(k^{\prime}\right) C_{p} .
$$

In the real situation, it is may be difficult to get the conditional probabilities. But in MPBS scheme, there is no necessity for the system to compute them, because the system only needs to page the following location areas in the descending order of $\alpha_{m+1}, \alpha_{m+2}, \cdots, \alpha_{k}$. Divided by a same positive number does not affect their order. The cost of $C_{p_{3}}$ is just like PBS scheme and the cost of $C_{p_{4}}$ is the same as IS-41/GSM MAP scheme. So we have

$$
C_{p_{3}}=E(k) C_{p},
$$

and

$$
C_{p_{4}}=C_{p}
$$

Thus, we can rewrite (3) as

$$
\begin{aligned}
C_{M P B S}= & \frac{\omega_{2} C_{u}}{\rho}+\left[\pi_{1}\left(1.5-Q\left(\frac{T_{\text {threshold }}}{\sigma}\right)\right)\right. \\
& \left.+\pi_{2} E\left(k^{\prime}\right)+\pi_{3} E(k)+\pi_{4}\right] C_{p} .
\end{aligned}
$$

\section{SimUlation RESUlTS AND COMPARISON}

In practical system, it is usually very difficult to derive $\omega_{1}, \omega_{2}$ and $\pi_{i}$ because they can be affected by many factors. In this section, we try to compare the scheme performances by simulations. In the simulations, we treat the state update message the same as the registration message and normalize the update cost $C_{u}=1$. The paging cost is usually less than the update cost, so we assume the paging cost for one location area is $0<C_{p} \leq C_{u}$. We are interested in how the CMR, the paging cost $C_{p}$, the user distribution probability $\alpha_{i}$ and the user out-of-pattern probability $\varphi$ can affect the performance gain of the PBS and MPBS schemes. In this paper, we assume there are three representative profile distributions: uniform, linear and exponential, which are commonly used in theoretical analysis ( [6]).

In these simulations, we assume the update cost for every location area is same. The simulations are event driven. In the initiation, a user is generated randomly in one location area and set in state 1 . The conditional probability of the user in state 1 , state 2 and state 3 are $0.8,0.15$ and 0.05 respectively. The user out-of-pattern probability $\varphi=0.1$. In order to study the out-of-pattern probability affection on the scheme performance, the $\varphi$ will change during the simulations. The user roams among 16 interconnected location areas randomly with exponential distributed residence time under different means ( [7]). When the user enters a location area, an interrupt is generated and one update messages is sent to the HLR or not according to the different schemes.

We can see that, in Fig.2, the update cost of MPBS scheme is less than half of that for IS-41/GSM MAP, and the CMR does not affect the performance dramatically. In wireless 


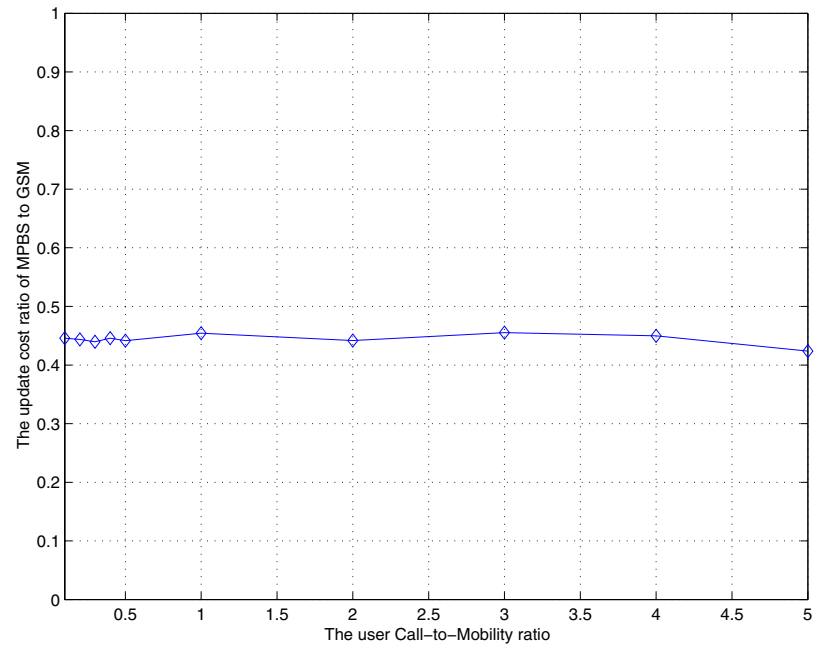

Fig. 2. The location update cost ratio of MPBS scheme to IS-41/GSM MAP

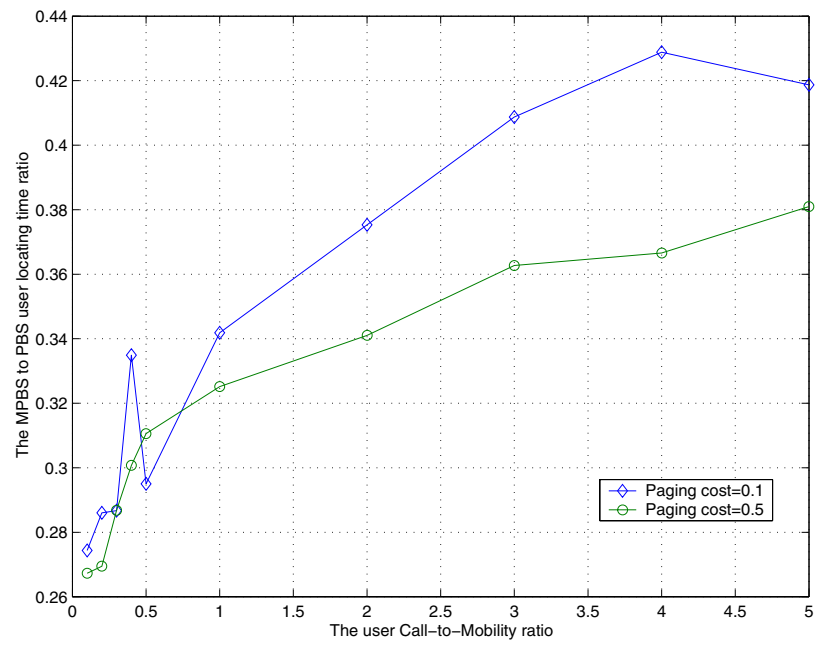

Fig. 3. The comparison of the paging delay for MPBS and PBS

communication system, the update cost is usually larger than the paging cost. That is why PBS and MPBS can achieve total saving by reducing the update cost at the expense of increasing the total paging cost. However the MPBS can reduce the total cost without increasing the total paging cost too much.

In both PBS and MPBS schemes, the system usually pages more than one location areas trying to find out the user's exact location. In other words, the MPBS and PBS will introduce some delay during call delivery procedures. In Fig.3, we can see that the paging delay generated by MPBS is much less than PBS. The reason is the follow. In the PBS scheme, all the location areas in the list are needed to be paged. Usually, the location areas are paged sequentially according to the user distribution probability. So the delay is the time elapsed from the paging messages is broadcasted in the first location area to the user responses is received. The delay can be different with different probability distributions. In Fig.3, we assume the user profile distribution is uniform. In the MPBS scheme, when the user is in-the-pattern, only when the user is in state

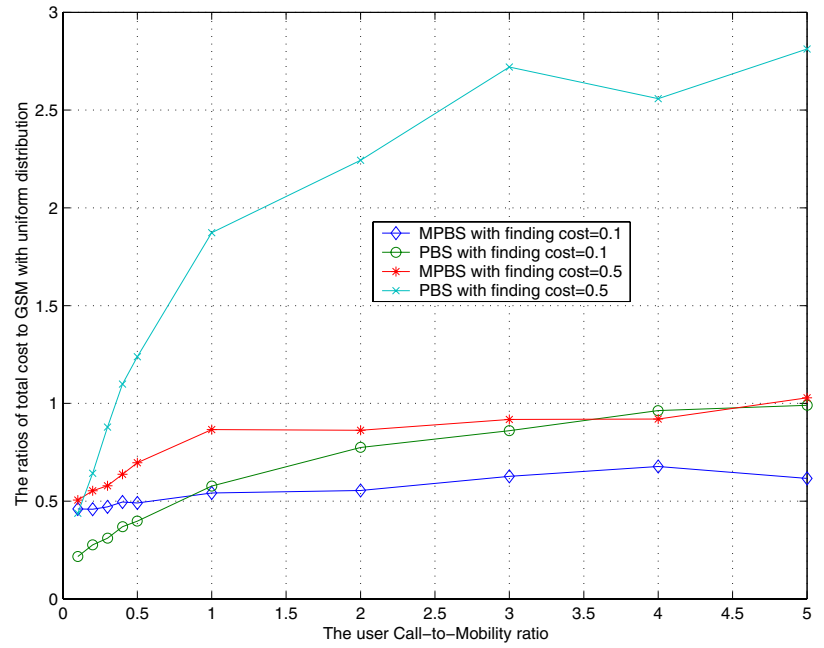

Fig. 4. The total costs of MPBS and PBS to IS-41/GSM MAP with uniform distribution

3 , need all location areas to be sequentially paged. When user is in state 1, only one location area is paged in most of time; when in state 2 , only part of the $\left\{A_{i}\right\}$ need to be paged. The paging cost could be considered as the paging delay for one location area. In our simulations, we assume the paging cost for every location area is equal. As we can see, when the CMR is low, the paging delay for MPBS scheme is $70 \%$ less than PBS scheme. The reason is that when the CMR is low, the user has relative high movement probability, the PBS scheme will page more location areas trying to find out the user; but the MPBS scheme is not affected by this factor. When the CMR is large, the user will stay in a location area for a relative long time, the PBS scheme can find out the user with less location area paging. However, the MPBS total paging cost is still $60 \%$ less than the PBS scheme.

Although the MPBS has less paging delay than PBS, we need find out the total costs for the two schemes and try to see whether they can achieve better performance than the conventional IS-41/GSM MAP scheme or not. In Fig.4, we plot both the MPBS and PBS relative costs to IS-41/GSM MAP under uniform distribution and different paging costs. We omit the linear and exponential distribution figures because of the space limit. The results are similar. In Fig.4, the cost of PBS scheme increases very quickly with the increase of paging cost. When the paging cost is 0.5 , the PBS scheme can have saving only when the CMR is very low. In that situation, the user will make a lot of updates to the system and the update cost is dominant. With the increase of CMR, the paging cost the for PBS scheme plays a more important role, then the total cost increases fast. The MPBS total cost increases much slowly. This is the advantage of MPBS. Another advantage of MPBS is that the cost curves are flatter than PBS in a wide range of CMR. That means the MPBS scheme is applicable to different classes of users with different mobility patterns. In the above situations, we assume in $90 \%$ of the time, the user is in-the-pattern. It is straightforward that the total costs 


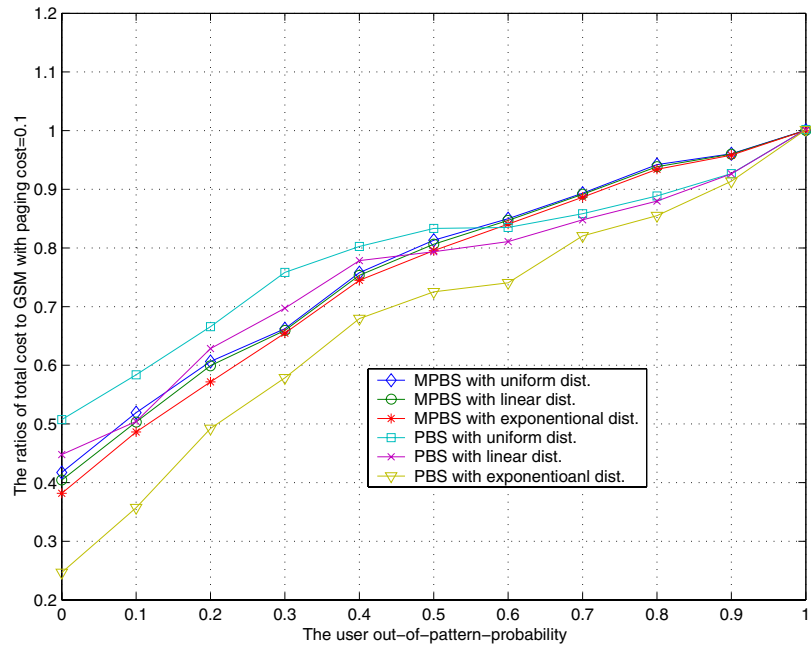

Fig. 5. The effects of user out-of-pattern probability on MPBS and PBS

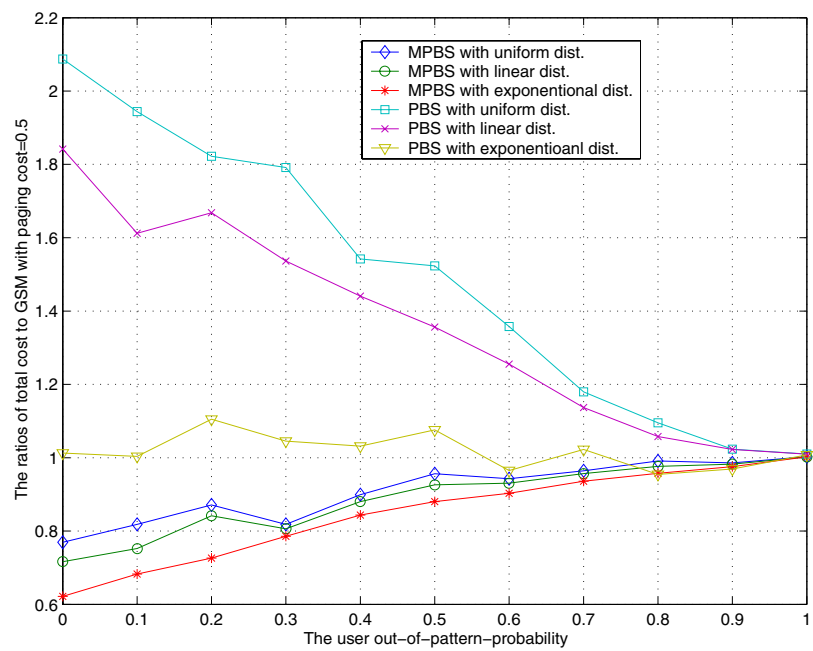

Fig. 6. The effects of user out-of-pattern probability on MPBS and PBS

of both MPBS and PBS schemes depend on the out-of-pattern probability greatly.

Fig.5 and Fig.6 show how the user out-of-pattern probability affects the system performance for both MPBS and PBS schemes with different probability distributions. In both figures, we assume $C M R=1$. The paging cost is 0.1 in Fig.5 and 0.5 in Fig.6. In Fig.5, both the PBS and MPBS schemes total costs increase as the user out-of-pattern probability increases. The cost of MPBS is less than PBS. When the paging cost is large, as in Fig.6, the total costs for PBS are larger than one for all the distributions. This proves that the PBS scheme can improve the system performance only in very limited conditions. In Fig.6, the total costs of MPBS are still less than one even the user has large probability to get out of the pattern. In the two figures, all the cost ratios are equal one when the user out-of-pattern probability is one. The reason is that when the user out-of-pattern is one, the user will not enter any location area in the profile list and needs to register to the system every time he/she moves. The system mobility management scheme is the same as IS-41/GSM MAP scheme indeed.

\section{CONCLUSION}

In this paper, we analyzed the PBS scheme and MPBS scheme by simulations. The results show that the system performance of PBS has tight relationship with the user's callto-mobility ratio. We simulated the PBS scheme performance based on different probability distributions, paging costs and CMR. The affection of the user's out-of-pattern probability is also studied. The results suggest that PBS scheme only works well for very small CMR and the total cost increases quickly with the paging cost. The simulation results also show that the total cost of MPBS is significantly less than PBS and the MPBS scheme is not very sensitive to the increase of paging cost either. Our simulation results show that the paging delay of MPBS scheme can be 50\% less than PBS scheme too.

\section{ACKNOWLEDGMENT}

This work was supported in part by National Science Foundation Faculty Early Career Development Award under grant ANI-0093241.

\section{REFERENCES}

[1] EIT/TIA, "Cellular radio telecommunications intersystem operations", Technical Report IS-41 (Revision B), EIA/TIA (1991).

[2] ETSI, Digital cellular telecommunications system (phase 2+): mobile application part (MAP) specification (GSM 09.02 version 7.51 Release), 1998.

[3] V.W.-S Wong and V. C. M. Leung, "Location Management for NextGeneration Personal Communications Networks", IEEE Network,pp.1824, September/October 2000.

[4] I.F. Akyildiz, J. McNair, J.S.M. Ho, H. Uzunalioglu and W. Wang "Mobility management in next-generation wireless systems", Proc. of the IEEE, vol.4, no.5, October 1996.

[5] S. Tabbane, "An Alternative Strategy for Location Tracking”, JSAC, vol.13, no. 5, June 1995

[6] G. P. Pollini and Chih-Lin I, "A Profile-Based Location Strategy and Its Performance", IEEE JSAC, vol.15, no.8, October 1997.

[7] W. Ma and Y. Fang, "A New Location Management Strategy Based on User Mobility Pattern for Wireless Networks", Proc. IEEE LCN 2002, Tampa, November, 2002. 